\title{
Bilateral posterior lamellar corneal transplant surgery in an infant of 17 weeks old: surgical challenges and the added value of intraoperative optical coherence tomography
}

\author{
Marc Muijzer ${ }^{1}$, Hester Y. Kroes ${ }^{2}$, Peter Van Hasselt ${ }^{2}$, and Robert Wisse ${ }^{1}$ \\ ${ }^{1}$ University Medical Center Utrecht \\ ${ }^{2}$ Wilhelmina Children's Hospital, University Medical Center Utrecht
}

November 1, 2021

\begin{abstract}
To describe the surgical challenges, management, and value of intra-operative optical coherence tomography in a case of a bilateral Descemet Stripping Automated Endothelial Keratoplasty corneal transplantation at 17 weeks of age for the treatment of severe posterior polymorphous corneal dystrophy resulting from a de novo mutation of the OVOL2-gene.
\end{abstract}

Bilateral posterior lamellar corneal transplant surgery in an infant of 17 weeks old: surgical challenges and the added value of intraoperative optical coherence tomography.

M.B. Muijzer ${ }^{1}$, H.Y. Kroes MD, $\mathrm{PhD}^{2}$, P.M. van Hasselt MD, $\mathrm{PhD}^{3}$, R.P.L. Wisse $\mathrm{MD}, \mathrm{PhD}^{1,4}$

${ }^{1}$ Department of Ophthalmology, University Medical Center Utrecht, Utrecht, the Netherlands

2 Department of clinical genetics, University Medical Center Utrecht, Wilhelmina Children's Hospital, Utrecht, the Netherlands

${ }^{3}$ Department of metabolic diseases, Wilhelmina Children's Hospital, University Medical Center Utrecht, Utrecht, the Netherlands.

4 Department of Ophthalmology, University Medical Center Utrecht, Wilhelmina Children's Hospital, Utrecht, the Netherlands

\section{Corresponding author}

M.B. Muijzer Utrecht Cornea Research Group, Department of Ophthalmology, University Medical Center Utrecht. Heidelberglaan 100, 3508 GX Utrecht, The Netherlands, Tel: +31 (0) 88755 1683; Fax: +31 (0) 88755 5417; Email: m.b.muijzer@umcutrecht.nl

\section{Funding and conflict of interest statement}

\section{Conflict statement:}

The authors have no financial or proprietary interest in the materials presented herein. MBM and RPLW are supported by the Dr. F.P. Fischer Foundation, facilitated by the Foundation Friends from the UMC Utrecht, and a unrestricted grant from Zeiss GmbH. The following authors have no financial disclosures: HYK, PEQMC, PMvH, PAWJFS, ESMV-K;

Funding: No funding or grant support.

Authorship: All authors attest that they meet the current ICMJE criteria for Authorship. 
Work presented as mystery case on Dutch Ophthalmic Society Meeting 2020 Groningen

\begin{abstract}
To describe the surgical challenges, management, and value of intra-operative optical coherence tomography in a case of a bilateral Descemet Stripping Automated Endothelial Keratoplasty corneal transplantation at 17 weeks of age for the treatment of severe posterior polymorphous corneal dystrophy resulting from a de novo mutation of the OVOL2-gene.
\end{abstract}

Key Words: endothelial keratoplasty, Descemet Stripping Automated Endothelial Keratoplasty, posterior polymorphous corneal dystrophy, intra-operative optical coherence tomography, juvenile corneal transplant, OVOL2 gene

\title{
Key Clinical message:
}

We present a severe case of PPCD resulting from a rare de novo pathogenic mutation of the OVOL2 gene warranting a bilateral corneal transplantation at a young age. The direct imaging of intra-operative OCT may aid surgery in severely clouded corneas.

\section{Introduction}

Posterior polymorphous corneal dystrophy (PPCD) is a rare autosomal dominant corneal endothelial dystrophy. The dystrophy is characterized by abnormal endothelial cell morphology, which appear as vesicular lesions, grey-white opacities, and linear bands during slit lamp examination. ${ }^{1}$ PPCD is genetically heterogeneous and can be caused by pathogenic mutations in the OVOL2 gene (PPCD1), COL8A2 gene (PPCD2), ZEB1 gene (PPCD3), and GRHL2 gene (PPCD4) ${ }^{1,2}$ In general, PPCD is considered a mild condition, where only a minority develops symptoms in infancy resembling a Congenital Hereditary Endothelial Dystrophy (CHED). These symptoms include: corneal edema, peripheral iridocorneal adhesions, and glaucoma. Of these symptoms, in particular corneal edema can have a huge impact on the visual development due to amblyopia. ${ }^{3}$

It is estimated about $20-25 \%$ of PPCD cases who develop corneal edema require corneal transplant surgery. ${ }^{4}$ In recent years endothelial keratoplasty is increasingly preferred as a first-choice treatment for corneal endothelial transplant surgery in children. ${ }^{5,6}$ Here, the structural integrity of the cornea is retained and a superior acuity is achieved. However, endothelial keratoplasty requires corneal clarity and as a consequence, standard endothelial keratoplasty, is not always feasible in cases with severe corneal clouding. To this end, intraoperative optical coherence tomography (iOCT) theoretically provides a solution. iOCT is a novel application of a widely used non-invasive in vivo imaging based on infrared light interferometry. The iOCT signal is typically not affected by corneal clouding and provides the surgeon with high resolution in-depth images of the surgical field. ${ }^{7}$

We present a case of a 4-month-old infant with PPCD1 and severe corneal clouding who was successfully treated with iOCT-assisted lamellar endothelial keratoplasty in both eyes. Our extensive reporting of the surgical challenges in this case and the description of the benefits of intra-operative OCT during surgery may help ophthalmologist in similar cases.

\section{Case description}

A newborn boy of 9 weeks old was referred with progressive clouding of both eyes. Slit lamp examination showed diffuse corneal clouding, a symmetrical cornea diameter of $11 \mathrm{~mm}$, and intact pupillary reflexes. No evident irido-corneal adhesions or correctopia were found. Sodium, chloride drop 5 times daily and non-steroid anti-inflammatory ocular drops 1 time daily were prescribed with no effect and subsequently discontinued after 2 months. The pregnancy was normal (G1P1) with a spontaneous vaginal delivery at 41 weeks gestational age. Physical examination was performed by the pediatrician and the clinical geneticist. In addition, extensive metabolic screening was performed. Both the physical examination and metabolic screening revealed no abnormalities related to the condition. A tentative diagnosis of CHED was made. Given the genetic heterogeneity of CHED/PPCD, genetic testing was performed by high throughput DNAanalysis and subsequent in silico analysis of 424 genes associated with visual impairment (consisting of 
single nucleotide variant and copy number variant analysis) followed by SNP-array. This revealed a de novo chromosomal duplication of $\sim 49 \mathrm{~Kb}$ in the region 20p11.23, encompassing the entire OVOL2 gene. The duplication was absent in DNA from the parents with no known family history of PPCD or consanguinity.

Additional ophthalmic examinations were performed under general anesthesia. The iOCT imaging revealed

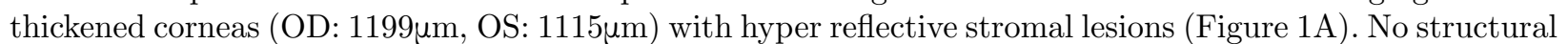
abnormalities of the anterior segment were found. The red reflex in mydriasis was absent in both eyes, though ultrasound echography showed no abnormalities in the posterior segment. The intraocular pressure (IOP) measured $18 \mathrm{mmHg}$ ODS and the axial length was $18.2 \mathrm{~mm}$ OD and $17.85 \mathrm{~mm}$ OS (conform his age).

Based on the identified pathogenic OVOL2 -duplication, the corneal clouding was diagnosed as PPCD1. The aggressive course of the disease would inevitably lead to severe visual impairment. With informed consent from both parents, and after consultation with our pediatric ophthalmologists and an international board of corneal specialists, we decided to perform a bilateral lamellar endothelial keratoplasty.

\section{Surgical treatment:}

At 17 weeks of age a bilateral Descemet Stripping Automated Endothelial Keratoplasty (DSAEK) corneal transplantation was performed in the Wilhelmina Children's hospital (Figure 2B). An $8.5 \mathrm{~mm}$ pre-cut organ cultured unmarked DSAEK tissue was provided by the eye bank (ETB-bislife, Beverwijk, the Netherlands). The graft endothelial cell density measured 2800 and 2600 cells $/ \mathrm{mm}^{2}$ and the graft thickness measured $133 \mu \mathrm{m}$ and $106 \mu \mathrm{m}$ for respectively the right eye and the left eye. Largely the same surgical procedure was subsequently used for both eyes. After creating two paracenteses nasally and temporally the surgeon performed a peripheral iridectomy at the 6 o'clock position. In the first procedure (OD) a Lewicky anterior chamber maintainer was used (DORC International, Zuidland, the Netherlands). In the left eye a viscoelastic device (Healon, Abbot Medical, Chicago, IL, USA) was used to maintain the anterior chamber and prevent IOP differences and rinsed carefully after graft positioning. ${ }^{8}$ A high vitreous pressure resulted in iris prolapse in both eyes, which was subsequently repositioned. Repositioning of the iris prolapse in the right eye proved challenging because of the high vitreous pressure, which resulted in an iris defect and damage to the sphincter muscle. Repositioning of the iris in the left eye was only possible after relieving the intraocular pressure by pars plana aspiration. The Descemet membrane was not attempted to be removed, as the Descemet membrane cannot be clearly identified in infants. ${ }^{5} \mathrm{~A} 4 \mathrm{~mm}$ corneal-scleral incision was made, and nylon 10-0 non-absorbable stitches (Ethicon, Sommersville, NJ, USA) were prepared. Prior to insertion and owing to the absent visualization, the graft was stained using membrane blue (DORC International, Zuidland, the Netherlands) and inserted using a Maculuso inserter (Janach Instruments, Como, Italy).

A microscope-integrated iOCT (OPMI Lumera 700; Carl Zeiss, Jena, Germany) was used during the surgery to visualize various steps during surgery, such as graft orientation, position, adherence, and interface (Figure 2B). Graft orientation was assessed using the acute-angled bevel sign described by Titiyal et al.; a sharp angle $\left(<90^{\circ}\right)$ between the graft' edge and recipient posterior corneal surface is indicates a correct orientation of the graft. ${ }^{9}$ The graft was fixated using a large air bubble and iOCT image revealed persistent interface fluid, which resolved after continuous corneal swiping. Afterwards, the iOCT image confirmed a fully adhered donor lenticule with no interface abnormalities and consequently no prolonged overpressure of the globe was applied. At the end of surgery, large air bubble was retained and the patient received a peribulbar injections of dexamethasone $4 \mathrm{mg} / \mathrm{ml}$. Postoperative medication included dexamethasone $0.1 \% /$ tobramycine $0.3 \%$ eye drops 6 times daily (Novartis Pharma, Amsterdam, the Netherlands) and prednisolone ointment ante noctem for 3 months (Ursapharm, Luik, Belgium). After this period the tobradex was switched to $0.1 \%$ dexamethasone eyedrops QID (Thea Pharma Benelux, Wetteren, Belgium).

\section{Outcome and follow-up}

One week after surgery the right eye started to clear, whereas the cornea of the left eye remained cloudy. Because of the young age of the patients and difficulty in examining the eye repeated postoperative examinations were performed under general anesthesia. .During re-examination 2 weeks post-operative iOCT showed a completely attached graft in the right eye and a $>75 \%$ detached graft in in the left eye, which was 
re-attached directly using a complete fill of the anterior chamber with air. Nonetheless, the cornea of the left eye remained cloudy after surgery and the boy developed an esotropia and horizontal pendular nystagmus. Another re-examination was performed 10 weeks after initial surgery. During this examination iOCT revealed completely attached grafts in both eyes (Figure 1C). The graft in the left eye was markedly thicker compared to the graft of the right eye, respectively $197 \mu \mathrm{m}$ vs. $85 \mu \mathrm{m}$, and remained cloudy indicating that the graft was non-functional. The IOP measured $24 \mathrm{mmHg}(\mathrm{OD})$ and $28 \mathrm{mmHg}(\mathrm{OS})$. Because the apparent dysfunction of the graft in the left eye a re-transplant was planned and IOP lowering medication was started (timolol/brinzolamide BID).

At 29 weeks old a re-DSAEK of the left eye was performed following the same surgical procedure as described for the initial surgery for the left eye. However, during the re-transplant procedure the surgeon used a complete Sulphur hexafluoride $20 \%$ fill and 10 minutes overpressure to improve graft adherence. In the weeks after the re-transplant the cornea of the left eye started to clear and both the nystagmus and esotropia decreased. Despite the left eye lacked a proper red reflex and the pupil did not respond to mydriatics for which pediatric cataract surgery was planned. During the surgery a pupillary fibrotic membrane with a clear lens was identified and the membrane could be removed without complications (Figure 2E). In addition, after accelerated tapering of steroids and switching $0.1 \%$ fluorometholone (Allergan Nederland BV, The Netherlands) the IOP normalized.

He exhibited a relative normal visual behavior, owing to the normal visual development in the right eye, whereas the left eye experienced a deprivation amblyopia. Despite the removal of the pupillary membrane, an intractable miosis persisted, and he did not have a proper red reflex in the left eye. Occlusive patching was commenced at 9 months of age, with mixed compliance. Importantly, orthoptic examination showed visual responses in the left eye and a decrease in the nystagmus and esotropia.

At 14 months the boy was again examined under general anesthesia. In both eyes the graft was attached and functional. In the right eye the cornea fully cleared, whereas the left cornea was relatively clear. The crystalline lens was clear in both eyes, though the left eye lacked a proper red reflex. The IOP normalized, with no apparent sequelae of the prolonged ocular hypertension. Funduscopic examination did not reveal abnormalities in the posterior segment. To date, at 20 months of age, both corneas remained clear. The right eye showed a promising visual potential, whereas he can use left eye for spatial orientation and the recognition of coarse objects.

\section{Discussion}

Here, we describe the clinical course of an infant boy with severe PPCD1 who was treated with bilateral iOCT-assisted DSAEK at 17 weeks of age. Imminent corneal blindness warranted this high-risk intervention at such a young age. After surgery corneal clarity was restored in the right eye and the boy exhibits a good visual functioning of his right eye. The left eye had a more prolonged surgical course, with eventually a functional graft was achieved after 4 surgical interventions, albeit with a suboptimal red reflex. He is currently treated for a deprivation amblyopia, with guarded hopes for the amelioration of visual function and isophoria of the left eye. The risk of secondary ocular hypertension remains present, since PPCD is an independent risk factor for the development of glaucoma, in addition to his steroid dependency. Furthermore, particularly interesting about this case is the non-hereditary mutation of the OVOL2 gene, while in the majority of the reported cases a hereditary link has been confirmed. ${ }^{1,2,4}$

Endothelial keratoplasty is increasingly preferred for the treatment of congenital endothelial disorders compared to penetrating keratoplasty. In children who underwent penetrating keratoplasty visual rehabilitation may be complicated because of amblyopia, suture-related complications, high astigmatism, graft rejection, and in the long-term graft failure. Nevertheless, performing endothelial keratoplasty in children is more challenging than in adults because of the smaller corneal diameter, higher scleral elasticity, shallower anterior chamber, and difficulty maintaining a supine position after surgery. Moreover, treatment is further complicated by difficulties in examination the eye. Only a few cases are reported to undergo surgery at an infant age. ${ }^{4-6,10-12}$ The reports of endothelial keratoplasty at infant age show in general a successful proce- 
dure. Notwithstanding, most reported cases developed a graft detachment in at least one eye which required re-bubbling of the graft.

The availability of the iOCT proves a crucial asset in the treatment of this case: without endothelial keratoplasty would have been challenging. The high-resolution imagery of the iOCT provided direct and accurate 3D spatial information about the cornea, anterior segment, and graft. In line with other studies we found that the availability of iOCT had a positive influence on critical decision-making during surgery and followup. ${ }^{7}$ The iOCT proved to be decisive in determining the orientation and confirming the adherence of the graft, which led to refraining from prolonged overpressure of the globe

In conclusion, we report the first bilateral iOCT-assisted DSAEK in an infant. At 20 months old he shows a remarkably normal visual behavior. We hope that reporting the extensive genetic and metabolic workup, description of the advantage of iOCT, and our judicious considerations helps other corneal surgeons in clinical decision making for these rare and high-risk cases.

\section{Patient Consent}

Consent to publish this case report has been obtained from both parents in writing.

Acknowledgement: We would like to thank P.E.Q.M. Cooijmans, P.A.W.J.F. Schellekens, and E.S.M. Voskuil-Kerkhof of the department of Ophthalmology of the University Medical Center Utrecht for their involvement in the treatment of this patient and L. Haer-Wigman of the Department of Human Genetics of the Radboud University Medical Center for her assistance in making the molecular diagnosis.

\section{Author Contributions:}

MM and RW: drafted the manuscript, MM, HK, PH, RW: critical revision of the manuscript. all authors: read and approved the final predraft manuscript.

\section{References:}

1. Davidson AE, Liskova P, Evans CJ, et al. Autosomal-Dominant Corneal Endothelial Dystrophies CHED1 and PPCD1 Are Allelic Disorders Caused by Non-coding Mutations in the Promoter of OVOL2. Am J Hum Genet . 2016;98(1):75-89. doi:10.1016/j.ajhg.2015.11.018

2. Liskova P, Dudakova L, Evans CJ, et al. Ectopic GRHL2 Expression Due to Non-coding Mutations Promotes Cell State Transition and Causes Posterior Polymorphous Corneal Dystrophy 4. Am J Hum Genet . 2018;102(3):447-459. doi:10.1016/j.ajhg.2018.02.002

3. Graham MA, Azar NF, Dana MR. Visual rehabilitation in children with congenital hereditary endothelial dystrophy. Int Ophthalmol Clin . 2001;41(4):9-18.

4. Hermina Strungaru M, Ali A, Rootman D, Mireskandari K. Endothelial keratoplasty for posterior polymorphous corneal dystrophy in a 4-month-old infant. Am J Ophthalmol Case Reports . 2017;7(2017):23-26. doi:10.1016/j.ajoc.2017.05.001

5. Madi S, Santorum P, Busin M. Descemet stripping automated endothelial keratoplasty in pediatric age group. Saudi J Ophthalmol . 2012;26(3):309-313. doi:10.1016/j.sjopt.2012.04.006

6. Busin M, Beltz J, Scorcia V. Descemet-stripping automated endothelial keratoplasty for congenital hereditary endothelial dystrophy. Arch Ophthalmol . 2011;129(9):1140-1146. doi:10.1001/archophthalmol.2011.114

7. Siebelmann S, Hermann M, Dietlein T, Bachmann B, Steven P, Cursiefen C. Intraoperative optical coherence tomography in children with anterior segment anomalies. Ophthalmology . 2015;122(12):25822584. doi:10.1016/j.ophtha.2015.06.004

8. Alqudah AA, Bauer AJ, Straiko MD, Sanchez JS, Terry MA. Descemet stripping automated endothelial keratoplasty. Medicine (Baltimore) . 2020;99(45):e23139. doi:10.1097/MD.0000000000023139 
9. Titiyal JS, Kaur M, Shaikh F, Bari A. "Acute-angled bevel" sign to assess donor lenticule orientation in ultra-thin descemet stripping automated endothelial keratoplasty. BMJ Case Rep . 2019;12(2). doi:10.1136/bcr-2018-227927

10. Bellucci R, Chierego C, Bellucci C. Endothelial keratoplasty in a newborn baby with CHED. Cornea . 2011;30(12):1488-1490. doi:10.1097/ICO.0b013e318221c2f3

11. Goshe JM, Li JY, Terry MA. Successful Descemet's stripping automated endothelial keratoplasty for congenital hereditary endothelial dystrophy in a pediatric patient. Int Ophthalmol . 2012;32(1):61-66. doi:10.1007/s10792-011-9511-3

12. Sella R, Rootman D, Bahar I. Descemet's stripping automated endothelial keratoplasty for posterior polymorphous corneal dystrophy in an 8-month-old boy. $J$ AAPOS . 2013;17(1):94-96. doi:10.1016/j.jaapos.2012.09.009

Figure legend:

Figure 1. Intraoperative OCT imaging In the upper image (A) is the right eye shown during initial examination and in the lower image (B) the right eye during primary surgery. In both images the left panel shows the en face microscope view, and in the right panel the corresponding live OCT image. The location and direction of the live OCT image are highlighted by the turquoise arrow in the white square in the left panel. In the upper image (A) significant corneal clouding can be seen, the OCT images shows hyper reflective lesions in the stroma compared to the surrounding tissue. The anterior chamber angle measures 47 degrees and no structural abnormalities. The middle image (B) shows the right eye during primary surgery. Because of severe corneal edema the graft is poorly visible in the en face microscope view. However, using the live OCT image the surgeon was able to clearly visualize the graft during surgery. The lower image (C) shows the right eye 10 weeks after primary surgery. The en face microscope view shows an clear cornea and bright red reflex. In the OCT image an attached and de-swelled graft can be observed.

Figure 2. Timeline of major examinations In Figure 2 we constructed an timeline of important events and examinations of this case. The left image shows the right eye (OD) and the right image the left eye (OS) at the time of the intervention described left of the images (e.g. first examination, primary surgery etc.). Right of the images the age of the boy is given at the time of the intervention. At the first examination (A) there is a significant amount of corneal edema at 12 weeks of age. The images at the primary surgery (B) show the increased severity of corneal edema and the difficulty to visualize the graft. Two weeks after primary surgery $(\mathrm{C})$ the graft in the left eye was found to have detached and was subsequently re-attached by injecting air in the anterior chamber. Two months after the primary surgery (D) the cornea of the right eye cleared and a red reflex is visible, the cornea of the left eye is still severely clouded and was shortly afterwards the left eye was re-grafted. At a proximally 10 months of age an examination with the use of mydriatics was performed. The right eye shows a clear cornea with a bright red reflex, whereas the left cornea is still hazy and shows no red reflex $(\mathrm{E})$. 
A: The right eye during the first examination under general anesthesia.

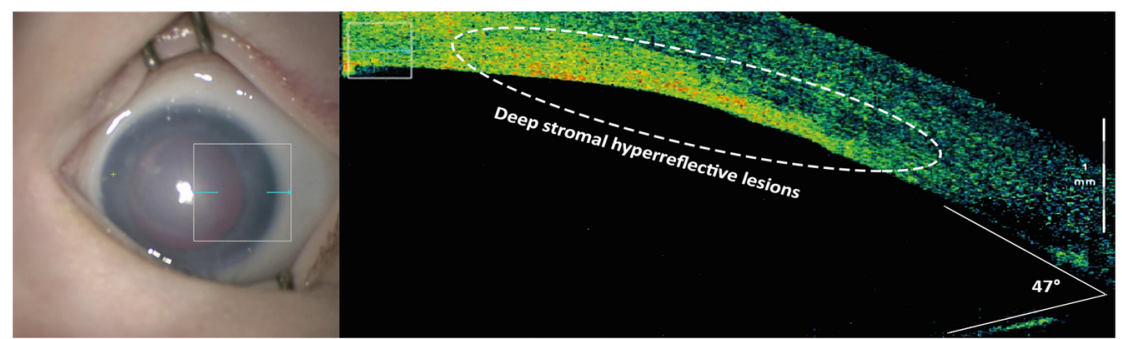

B: The right eye during primary surgery ( 5 weeks later)

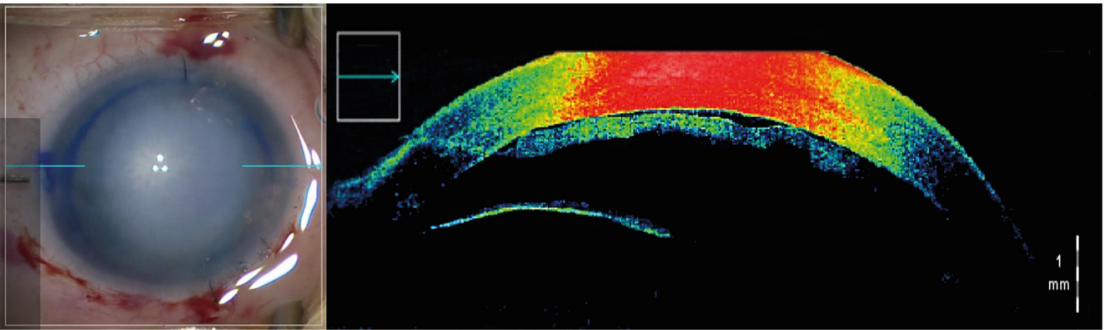

C: The right eye 10 weeks after primary surgery

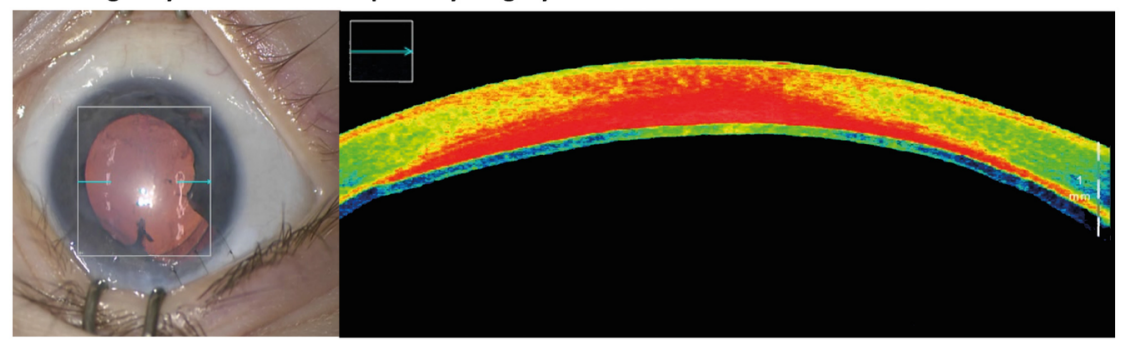




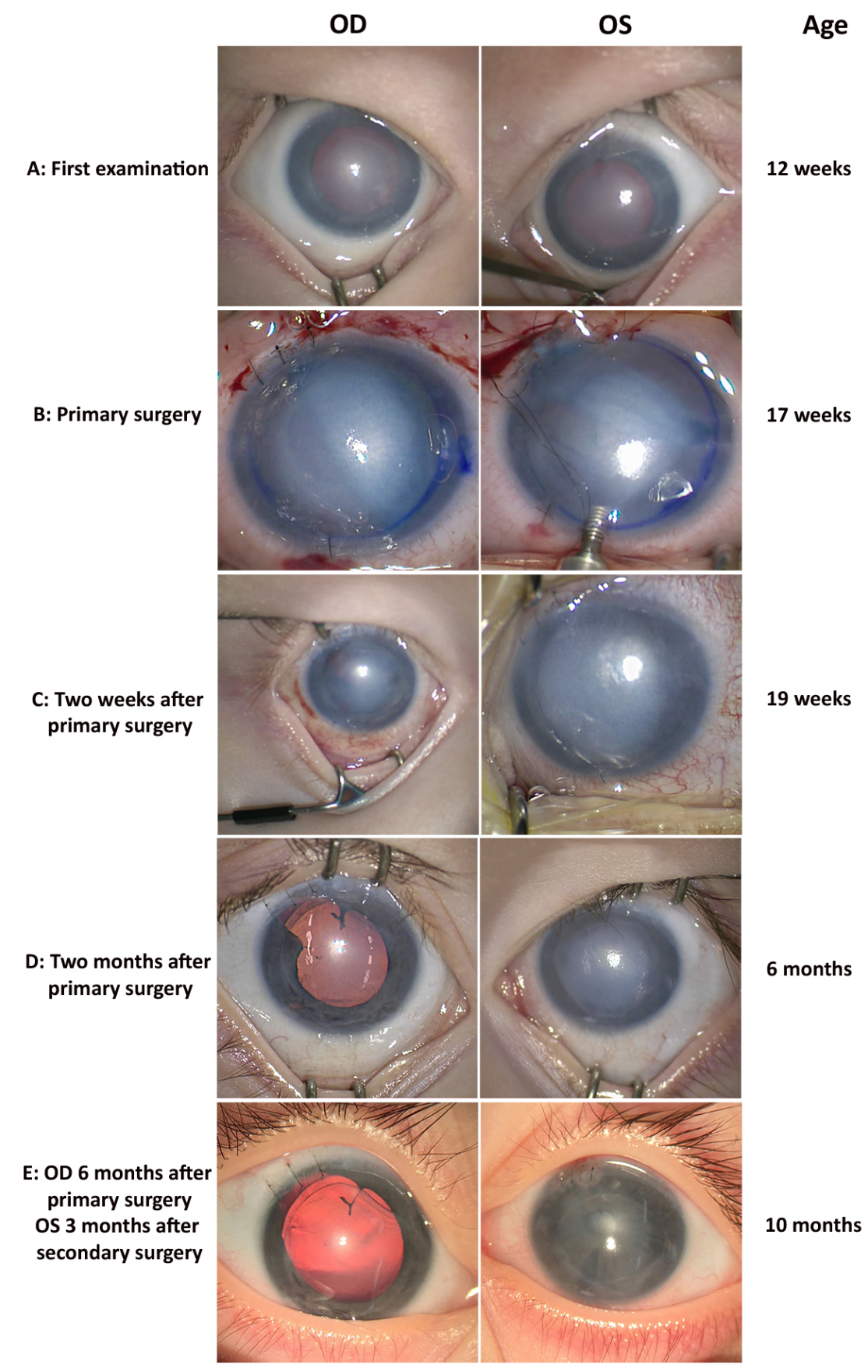

